\title{
Multivariate Analysis of Tronchuda Cabbage (Brassica oleracea L. var. costata DC) Phenolics: Influence of Fertilizers
}

\author{
Carla Sousa, ${ }^{\dagger}$ David M. Pereira,${ }^{\dagger}$ José A. Pereira, ${ }^{\S}$ Albino Bento, ${ }^{\S}$ \\ M. Angelo Rodrigues, ${ }^{\S}$ Sonia Dopico-García, ${ }^{\dagger}$ Patrícia Valentão, ${ }^{\dagger}$ \\ Graciliana Lopes, ${ }^{\dagger}$ Federico Ferreres, ${ }^{\#}$ Rosa M. Seabra, ${ }^{\dagger}$ And \\ PAUla B. ANDRADE $*,+$ \\ REQUIMTE/Serviço de Farmacognosia, Faculdade de Farmácia, Universidade do Porto, R. Aníbal \\ Cunha, 164, 4050-047 Porto, Portugal; CIMO/Escola Superior Agrária, Instituto Politécnico de \\ Bragança, Campus de Sta Apolónia, Apartado 1172, 5301-855 Bragança, Portugal; and Research \\ Group on Quality, Safety and Bioactivity of Plant Foods, Department of Food Science and \\ Technology, CEBAS (CSIC), P.O. Box 164, 30100 Campus University of Espinardo (Murcia), Spain
}

\begin{abstract}
A field experiment was carried out to investigate the effect of fertilization level on the phenolic composition of tronchuda cabbage (Brassica oleracea L. var. costata DC) external and internal leaves. Eight different plots were constituted: a control without fertilization, one with organic matter, and six experiments with conventional fertilizers (nitrogen, boron, and sulfur, two levels each). The phenolic compounds were analyzed by reversed-phase HPLC-DAD. External and internal leaves revealed distinct qualitative composition. In the internal leaves were found 15 phenolics (5 kaempferol and 10 cinnamic acid derivatives), whereas the external leaves presented 3-p-coumaroylquinic acid and 13 kaempferol derivatives. Principal component analysis (PCA) was applied to assess the relationships between phenolic compounds, agronomical practices, and harvesting time. Samples obtained with conventional practices were quite effectively separated from organic samples, for both types of leaves. In general, samples developed without any fertilization presented the highest phenolics amounts: external and internal leaves contained 1.4- and 4.6-fold more phenolic compounds than the ones that received conventional fertilizer, respectively, and the internal leaves presented 2.4 times more phenolics than the ones grown with organic amendment. Additionally, samples from organic production exhibited higher total phenolics content than those from conventional practices, collected at the same time. Samples harvested first were revealed to be distinct from the ones collected later. The results show that it is possible to grow tronchuda cabbage without excess fertilizers, with highest amounts of phenolics and reduced environment contamination.
\end{abstract}

KEYWORDS: Brassica oleracea L. var. costata DC; tronchuda cabbage; phenolic compounds; organic fertilization; nitrogen; boron; sulfur; principal component analysis

\section{INTRODUCTION}

It is well established that a greater daily intake of vegetables and fruits is associated with a smaller risk of the major deadly diseases in Western society. Plant secondary metabolites are the most likely candidates for this general health-promoting effect (1). Epidemiological studies indicate that consumption of cruciferous vegetables is more strongly associated with decreased cancer risk than fruit and vegetable consumption in

* Author to whom correspondence should be addressed (telephone + 351 222078935; fax + 351222003977 ; e-mail pandrade@ff.up.pt).

†EQUIMTE.

$\S$ CIMO.

${ }^{\#}$ CEBAS (CSIC). general, which can be attributed to the presence of sulfurcontaining compounds (2). Other phytochemicals, such as phenolic compounds, may also contribute to this effect.

Tronchuda cabbage (Brassica oleracea $\mathrm{L}$. var. costata DC), mainly consumed for its leaves, has already been characterized in terms of glucosinolates $(3,4)$ and phenolics $(5,6)$. Although genetics and seasonally induced changes are the primary determinants of the composition of secondary plant metabolites, the phenolics content may also be affected by environmental factors and the fertilization regimen $(7,8)$.

Soil mineral nitrogen availability may vary according to the amount of applied nitrogen and crop nitrogen uptake pattern (9). Nitrogen uptake, which is genetically determined, in Brassicas is higher than in many other food crops. Despite this, 


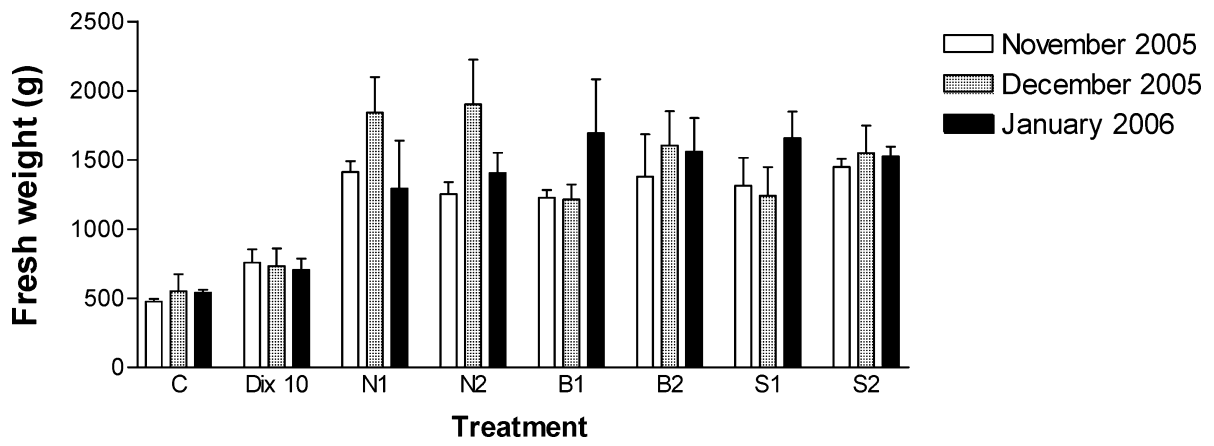

Figure 1. Fresh weight of $B$. oleracea var. costata plants subjected to different fertilization regimens. Results are expressed as means of three replicates, and standard deviation bars are on top of each column. C, control; Dix10, organic amendment; N1, $80 \mathrm{~kg}$ of N/ha; N2, $160 \mathrm{~kg}$ of N/ha; B1, $2.2 \mathrm{~kg}$ of B/ha; B2, $4.4 \mathrm{~kg}$ of B/ha; S1, $37.3 \mathrm{~kg}$ of S/ha; S2, $74.6 \mathrm{~kg}$ of S/ha.

a significant part of the nitrogen taken up is lost to the soil in dead leaves, during the growth cycle (10).

In contrast to conventional systems, in which synthetic fertilizers containing directly available inorganic nitrogen are used, organic systems rely on the activity of a diverse soil ecosystem to make nitrogen available to plants $(11,12)$. It has been shown that nitrogen can become a growth-limiting nutrient in organic production, affecting negatively yield (12). This problem is overcome by conventional farmers, who obtain roughly the same nutrient supply across a variety of soil types and farming systems, because fertilization is adjusted to the level that gives the highest yield. On the other hand, conventional farming systems are often associated with problems such as nitrate leaching and groundwater pollution, degradation of soil structure, decreased surface infiltration of water, and pesticide contamination $(9,13)$. In fact, the lower nitrogen mineralization rate in organic farming system may improve nutrient recycling and reduce the risk for nitrogen leaching and groundwater pollution $(1,9)$.

The production of phenolic metabolites responds to changes in nutrient availability in a highly complex manner (14). Nitrogen stress triggers the gene expression of flavonoid pathway enzymes, and nitrate availability was shown to directly affect the enzyme activity in the phenylpropanoid pathway $(7,15-$ 17). Some authors propose that variation in concentrations of secondary metabolites may be due to differences in plant growth and accumulation of biomass (14). Others noticed that some phenolics, such as kaempherol and quercetin, do not seem to be affected by nutrition levels to the same extent as plant growth $(16,18)$.

Boron is required for normal growth and development of all higher plants, and it is thought to be involved in three main aspects of plant physiology: structure of cell walls, membrane function, and metabolic activities (19). This nutrient is one of those responsible for the changes in concentration and metabolism of phenolic compounds in vascular plants, due to its effects on polyphenol oxidase activity (20). This enzyme is normally bound to membranes or walls in a latent form and becomes active when released under boron-deficient conditions, which leads to phenolic compounds accumulation (19).

Sulfur is often referred to as the fourth major plant nutrient, as it is an essential component of important metabolic and structural compounds (21). Whereas nitrogen is mainly used for structural macromolecules, sulfur plays critical roles in the catalytic or electrochemical functions of the biomolecules in cells. Sulfur is found in amino acids, oligopeptides, vitamins and cofactors, and a variety of secondary compounds, such as glucosinolates in the Brassicaceae $(2,22)$.
Although vegetables or vegetable food commodities are produced under conventional practices, there has been increasing interest in organic products. Consumer perception is that these products are healthier than the conventional ones, with higher nutritional value and reduced pesticide residues, with the advantage of being produced in a sustainable agricultural practice less harmful to the environment $(7,12,23)$. When several quality aspects of organically and conventionally grown plant-derived foods were compared, only small and inconsistent differences were found (1). In previous works comparing organic or conventional production, B. oleracea var. costata grown under organic practices showed a slight tendency to increased phenolics levels $(5,6)$, although it was not possible to conclude that this corresponds to higher antioxidant potential (24). However, this tendency was not evident for other Brassica species (23).

Due to the important role of phenolic compounds as health protective agents, the aim of this work was to evaluate the influence of fertilizers, namely, organic (Dix10) and chemicals (nitrogen, boron, and sulfur), on B. oleracea var. costata phenolic composition and biomass production. In addition, the interference of the level of chemical fertilizer was also checked.

\section{MATERIALS AND METHODS}

Standards and Reagents. $p$-Coumaric and sinapic acids were from Sigma (St. Louis, MO) and kaempferol 3-O-glucoside and kaempferol 3-O-rutinoside from Extrasynthése (Genay, France). Analytical grade formic and acetic acids were purchased from Merck (Darmstadt, Germany). The water was treated in a Milli-Q water purification system (Millipore, Bedford, MA).

Plant Material and Treatments. Tronchuda cabbage (B. oleracea var. costata) plants were grown under different fertilization regimes. The experimental work was carried out in one field located in Bragança, northeastern Portugal $\left(41^{\circ} 48^{\prime} \mathrm{N}, 6^{\circ} 44^{\prime} \mathrm{W}\right)$. The field had an inclination inferior to $5 \%$ and was turned up to the northeast. Sowing occurred in the middle of June 2005 , in a greenhouse $\left(22 \pm 2{ }^{\circ} \mathrm{C}, 80 \%\right.$ humidity). Young plants were transplanted to the field at the end of August, spaced at $0.8 \times 0.5 \mathrm{~m}$ between and within rows. Before the fertilization treatments, the soil was loamy textured with $0.83 \%$ organic matter, a $\mathrm{pH}\left(\mathrm{H}_{2} \mathrm{O}\right)$ of 5.2, and median phosphorus (54 mg of $\left.\mathrm{P}_{2} \mathrm{O}_{5} / \mathrm{kg}\right)$ and high potassium $\left(126 \mathrm{mg}\right.$ of $\mathrm{K}_{2} \mathrm{O} / \mathrm{kg}$ ) levels. Eight treatments were established: a control (C), without any fertilization, and one with Dix 10 (Crimolara, Portugal), an authorized organic amendment ( $10 \%$ total $\mathrm{N}, 3 \% \mathrm{~K}_{2} \mathrm{O}$, $3 \% \mathrm{P}_{2} \mathrm{O}_{5}, 2.5 \% \mathrm{CaO}, 0.6 \% \mathrm{MgO}$, and $30.5 \mathrm{mg}$ of $\mathrm{B} / \mathrm{kg}$ ); $\mathrm{N} 1$ and $\mathrm{N} 2$, with 80 and $160 \mathrm{~kg}$ of N/ha (80 kg/ha soil application $+80 \mathrm{~kg} / \mathrm{ha}$ side dress application in mid-October), respectively; B1 and B2, with 2.2 and $4.4 \mathrm{~kg}$ of $\mathrm{B} / \mathrm{ha}$, respectively; and $\mathrm{S} 1$ and $\mathrm{S} 2$, with 37.3 and 74.6 $\mathrm{kg}$ of $\mathrm{S} / \mathrm{ha}$, respectively. All conventional fertilizer regimens received $80 \mathrm{~kg}$ of N/ha to ensure plant growth, with the exception of the N2 
treatment. The fertilizers were simultaneously applied at the beginning of the growth season. The conventional fertilizers used were urea, borax, and magnesium sulfate (ADP, Portugal). Phosphorus (150 kg of super 18/ha) and potassium (50 $\mathrm{kg}$ of $\mathrm{KCl} / \mathrm{ha}$ ) were also used in these fields. Twelve cabbages were planted for each experimental treatment.

Samples were collected on three occasions during the growth season: in mid-November 2005 (a), mid-December 2005 (b), and mid-January 2006 (c). At each harvesting date and for each fertilization regimen three plants were randomly collected from three different plots. All samples were collected in the morning, at the same hour. After harvesting, the plants were immediately transported to the laboratory and weighed, and external and internal leaves were separated. Care was taken to choose plants and leaves of similar developmental stage: internal leaves, looking pale yellow and tender, were separated from the external ones, which presented a dark green color and were no longer actively expanding, although not yet senescent.

Each analyzed sample corresponds to the mixture of the three plants developed and collected in the same conditions. Two hours maximum after their collection, the samples were frozen at $-20{ }^{\circ} \mathrm{C}$ and then lyophilized (Labconco 4.5 Freezone apparatus, Kansas City, MO). The freeze-dried samples were powdered and kept in a desiccator in the dark, until they were subjected to phenolics extraction. To evaluate the dry weight, three plants of each treatment of the third harvesting time were dried at $65{ }^{\circ} \mathrm{C}$ until constant weight (6 days).

Phenolic Compounds Extraction. An aqueous extract was used for the phytochemical characterization: ca. $3.0 \mathrm{~g}$ of powdered $B$. oleracea var. costata leaves was boiled for $15 \mathrm{~min}$ in $300 \mathrm{~mL}$ of water and then filtered over a Büchner funnel. The resulting extract was lyophilized, and a yield of ca. $1.4 \mathrm{~g}$ was obtained. The lyophilized extract was kept in a desiccator, in the dark. The lyophilized extract was redissolved in water, in triplicate, immediately before the HPLCDAD analysis.

HPLC-DAD Quantitative Analysis of Phenolics. As internal and external leaves exhibit distinct phenolics composition $(5,25)$, to achieve a better separation of the compounds the HPLC gradients used for the analysis of the two kinds of leaves were different. The analysis of phenolic compounds was carried out as previously reported $(5,25)$ using a HPLC unit (Gilson) and a $250 \times 4.6 \mathrm{~mm}$ i.d., $5 \mu \mathrm{m}$ Spherisorb ODS2 column (Waters, Milford, MA), protected with a $4 \times 4 \mathrm{~mm}$ Spherisorb ODS2 guard column. For the internal leaves analysis, elution was performed using acetic acid 1\% (A) and methanol (B) as solvents, starting with $20 \%$ B and using a gradient to obtain $50 \%$ B at 30 min, $80 \% \mathrm{~B}$ at $37 \mathrm{~min}$, and $100 \% \mathrm{~B}$ at $42 \mathrm{~min}$. For the external leaves the solvent system was a mixture of formic acid 5\% (A) and methanol (B), with the following gradient: 0 min, $10 \% \mathrm{~B} ; 25 \mathrm{~min}, 20 \% \mathrm{~B} ; 40$ min, $50 \%$ B; $45 \mathrm{~min}, 50 \%$ B; $46 \mathrm{~min}, 90 \% \mathrm{~B} ; 50 \mathrm{~min}, 90 \% \mathrm{~B} ; 55 \mathrm{~min}$, $100 \% \mathrm{~B} ; 58 \mathrm{~min}, 100 \% \mathrm{~B}$; and $60 \mathrm{~min}, 10 \% \mathrm{~B}$. The flow rate was 1 $\mathrm{mL} / \mathrm{min}$, and the injection volume was $20 \mu \mathrm{L}$. Detection was achieved with a Gilson diode array detector. Spectral data from all peaks were accumulated in the range of $200-400 \mathrm{~nm}$, and chromatograms were recorded at $330 \mathrm{~nm}$. The data were processed on Unipoint system software (Gilson Medical Electronics, Villiers le Bel, France). Peak purity was checked by the software contrast facilities.

Phenolic Compounds identification was based on the retention time and UV spectra of each peak, in comparison with data published before for external (5) and internal (25) B. oleracea var. costata leaves. Quantification was achieved by the absorbance recorded in the chromatograms relative to external standards. With the exception of kaempferol 3-O-glucoside, which was quantified as itself, the kaempferol derivatives were quantified as kaempferol 3-O-rutinoside, 3- and 4 - $p$-coumaroylquinic acids were quantified as $p$-coumaric acid, and sinapic acid derivatives were quantified as sinapic acid, because none of them was commercially available.

Statistical Analysis. Principal component analysis (PCA) was applied to the results. PCA was performed by the SPSS program (version 15.0). PCA was applied separately for external and internal leaves. Individual concentrations of each phenolic compound and fresh weight of the plant were selected as variables.
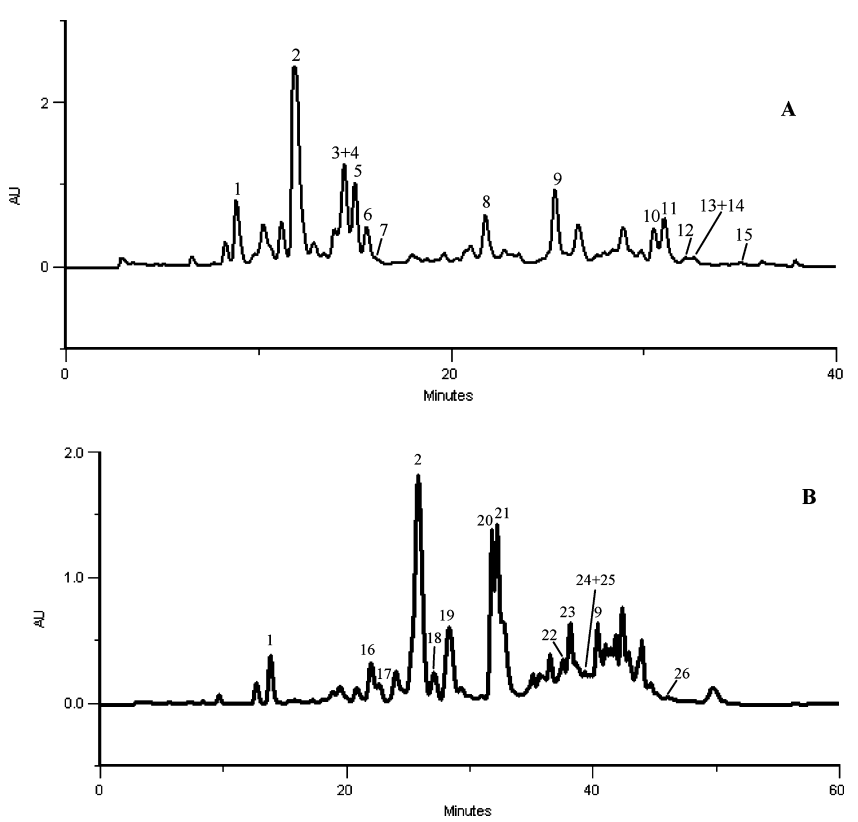

Figure 2. HPLC-DAD chromatogram of phenolic compounds of an aqueous extract of $B$. oleracea var. costata (control sample from the first harvesting): (A) internal leaves; (B) external leaves. Detection at $330 \mathrm{~nm}$. Compounds: (1) 3-p-coumaroylquinic acid; (2) kaempferol 3-O-sophoroside-7-O-glucoside; (3) sinapoyl glucoside acid; (4) kaempferol 3-O-(sinapoyl)-sophoroside-7-O-glucoside; (5) kaempferol 3-0-(feruloyl)sophoroside-7-O-glucoside; (6) kaempferol 3-O-(p-coumaroyl)-O-sophoroside-7-O-glucoside; (7) 4-p-coumaroylquinic acid; (8) sinapic acid; (9) kaempferol 3-O-sophoroside; (10) 1,2-disinapoylgentiobioside; (11) 1-sinapoyl-2-feruloylgentiobiose; (12) isomer of 1,2-disinapoylgentiobioside; (13) 1,2,2'-trisinapoylgentiobiose; (14) 1,2'-disinapoyl-2-feruloylgentiobiose; (15) isomer of 1,2-disinapoylgentiobioside; (16) kaempferol 3-0sophorotrioside-7-O-glucoside; (17) kaempferol 3-0-(methoxycaffeoyl/ caffeoyl)sophoroside-7-O-glucoside; (18) kaempferol 3-O-sophorotrioside7-O-sophoroside; (19) kaempferol 3-O-sophoroside-7-O-sophoroside; (20) kaempferol 3-O-(sinapoy//caffeoyl)sophoroside-7-O-glucoside; (21) kaempferol 3-O-(feruloyl/caffeoyl)sophoroside-7-O-glucoside; (22) kaempferol 3-Osophorotrioside; (23) kaempferol 3-0-(sinapoyl)sophoroside; (24) kaempferol 3-O-(feruloyl)sophorotrioside; (25) kaempferol 3-O-(feruloyl)sophoroside; (26) kaempferol 3-O-glucoside.

\section{RESULTS AND DISCUSSION}

Changes in Biomass. Tronchuda cabbages grown with no fertilization presented the lowest fresh weight, at all harvesting dates (Figure 1): on average, samples developed under organic amendment or chemical fertilization exhibited 1.4- and 2.8-fold the weight of those grown with no fertilization, respectively. Additionally, samples developed under organic amendment exhibited lower weight than the ones grown with traditional fertilization. Phenological development is driven by temperature and by photoperiod: a short photoperiod is associated with reduced peak radiation, reduced daily radiation, lower mean temperature, and lower minimum temperature. Of these, low minimum temperature would advance phenology in thermal time, whereas both short photoperiod and low radiation could have the reverse effect of slowing phenological development in Brassica species (26). Although a period of 3 months was considered, the harvesting time did not have a relevant effect on fresh weight in any of the samples.

All plants presented similar moisture contents, ranging from ca. 84 to $88 \%$, with no important differences among samples grown under different agronomic practices. 
Table 1. Quantification of Brassica oleracea var. costata Internal Leaves Phenolic Compounds (Milligrams per Kilogram, Dry Basis) ${ }^{a}$

\begin{tabular}{|c|c|c|c|c|c|c|c|c|c|c|c|c|c|c|c|}
\hline treatment & harvest $^{b}$ & \multicolumn{14}{|c|}{ phenolic compound } \\
\hline C & a & $\begin{array}{c}1263.6 \\
16.72\end{array}$ & $\begin{array}{l}8977.2 \\
101.70\end{array}$ & $\begin{array}{r}3785.8 \\
18.05\end{array}$ & $\begin{array}{c}2718.5 \\
44.57\end{array}$ & $\begin{array}{r}1413.2 \\
6.77\end{array}$ & $\begin{array}{c}288.6 \\
21.97\end{array}$ & $\begin{array}{r}911.8 \\
7.94\end{array}$ & $\begin{array}{c}4035.6 \\
100.02\end{array}$ & $\begin{array}{r}541.1 \\
\quad 1.70\end{array}$ & $\begin{array}{c}813.5 \\
1.92\end{array}$ & $\begin{array}{c}164.4 \\
4.03\end{array}$ & $\begin{array}{r}249.3 \\
4.80\end{array}$ & $\begin{array}{r}126.6 \\
0.55\end{array}$ & 25289 \\
\hline Dix10 & a & $\begin{array}{l}961.6 \\
20.80\end{array}$ & $\begin{array}{l}5709.4 \\
131.67\end{array}$ & $\begin{array}{c}4008.9 \\
105.12\end{array}$ & $\begin{array}{r}1453.4 \\
20.79\end{array}$ & $\begin{array}{l}992.6 \\
48.33\end{array}$ & $\begin{array}{c}74.6 \\
6.13\end{array}$ & $\begin{array}{r}547.1 \\
0.32\end{array}$ & $\begin{array}{r}1839.1 \\
38.76\end{array}$ & $\begin{array}{r}355.0 \\
0.57\end{array}$ & $\begin{array}{l}383.8 \\
4.29\end{array}$ & $\begin{array}{c}37.5 \\
1.99\end{array}$ & $\begin{array}{c}41.1 \\
0.87\end{array}$ & $\begin{array}{c}38.0 \\
3.53\end{array}$ & 16442 \\
\hline N1 & a & $\begin{array}{r}473.7 \\
4.60\end{array}$ & $\begin{array}{l}1615.8 \\
17.04\end{array}$ & $\begin{array}{r}1154.4 \\
5.26\end{array}$ & $\begin{array}{c}486.3 \\
3.16\end{array}$ & $\begin{array}{r}199.4 \\
2.33\end{array}$ & $\begin{array}{c}39.4 \\
0.72\end{array}$ & $\begin{array}{l}391.1 \\
1.24\end{array}$ & $\begin{array}{c}474.4 \\
9.02\end{array}$ & $\begin{array}{r}394.4 \\
4.59\end{array}$ & $\begin{array}{r}286.8 \\
8.36\end{array}$ & $\begin{array}{r}102.7 \\
1.55\end{array}$ & $\begin{array}{r}133.3 \\
2.45\end{array}$ & $\begin{array}{c}29.1 \\
1.63\end{array}$ & 5781 \\
\hline B1 & a & $\begin{array}{c}966.3 \\
60.91\end{array}$ & $\begin{array}{l}1262.5 \\
94.14\end{array}$ & $\begin{array}{r}954.7 \\
7.56\end{array}$ & $\begin{array}{l}414.0 \\
6.71\end{array}$ & $\begin{array}{r}178.4 \\
4.93\end{array}$ & $\begin{array}{c}107.6 \\
0.75\end{array}$ & $\begin{array}{c}432.6 \\
51.85\end{array}$ & $\begin{array}{c}666.2 \\
9.94\end{array}$ & $\begin{array}{c}437.5 \\
11.75\end{array}$ & $\begin{array}{l}362.5 \\
14.92\end{array}$ & $\begin{array}{l}99.7 \\
1.97\end{array}$ & $\begin{array}{c}103.3 \\
14.52\end{array}$ & $\begin{array}{l}7.5 \\
2.24\end{array}$ & 5993 \\
\hline B2 & a & $\begin{array}{c}610.6 \\
17.34\end{array}$ & $\begin{array}{l}2444.5 \\
21.70\end{array}$ & $\begin{array}{c}1156.6 \\
71.89\end{array}$ & $\begin{array}{l}810.7 \\
6.78\end{array}$ & $\begin{array}{l}240.9 \\
4.81\end{array}$ & $\begin{array}{c}79.4 \\
1.36\end{array}$ & $\begin{array}{c}346.9 \\
14.15\end{array}$ & $\begin{array}{c}1034.9 \\
15.45\end{array}$ & $\begin{array}{l}296.7 \\
5.55\end{array}$ & $\begin{array}{c}274.5 \\
0.59\end{array}$ & $\begin{array}{c}50.3 \\
1.37\end{array}$ & $\begin{array}{c}78.0 \\
1.33\end{array}$ & $\begin{array}{c}21.5 \\
0.97\end{array}$ & 7446 \\
\hline S1 & a & $\begin{array}{c}547.6 \\
14.29\end{array}$ & $\mathrm{nq}$ & $\begin{array}{c}353.9 \\
10.87\end{array}$ & $\begin{array}{c}83.0 \\
3.66\end{array}$ & $\begin{array}{c}60.3 \\
1.65\end{array}$ & $\begin{array}{c}49.6 \\
1.59\end{array}$ & $\begin{array}{c}191.8 \\
5.21\end{array}$ & $\begin{array}{c}649.5 \\
22.23\end{array}$ & $\begin{array}{c}25.3 \\
1.22\end{array}$ & $\begin{array}{c}18.3 \\
1.28\end{array}$ & $\begin{array}{c}11.2 \\
0.16\end{array}$ & $\begin{array}{c}22.4 \\
1.68\end{array}$ & $\begin{array}{l}26.6 \\
2.04\end{array}$ & 2040 \\
\hline Dix10 & b & $\begin{array}{l}527.0 \\
19.20\end{array}$ & $\begin{array}{l}1935.9 \\
29.62\end{array}$ & $\begin{array}{r}1884.8 \\
42.75\end{array}$ & $\begin{array}{c}631.1 \\
13.53\end{array}$ & $\begin{array}{r}144.5 \\
6.30\end{array}$ & $\mathrm{nq}$ & $\begin{array}{l}238.8 \\
6.48\end{array}$ & $\begin{array}{r}1332.3 \\
18.46\end{array}$ & $\begin{array}{r}184.7 \\
3.87\end{array}$ & $\begin{array}{c}172.1 \\
0.32\end{array}$ & $\begin{array}{c}50.6 \\
0.64\end{array}$ & $\begin{array}{r}106.8 \\
6.86\end{array}$ & $\begin{array}{c}15.2 \\
0.97\end{array}$ & 7224 \\
\hline N1 & b & $\begin{array}{l}262.8 \\
10.17\end{array}$ & $\begin{array}{l}279.1 \\
8.49\end{array}$ & $\begin{array}{r}246.3 \\
0.67\end{array}$ & $\begin{array}{c}33.2 \\
4.05\end{array}$ & $\begin{array}{c}37.0 \\
0.18\end{array}$ & $\begin{array}{c}34.6 \\
1.38\end{array}$ & $\begin{array}{c}195.6 \\
10.59\end{array}$ & $\begin{array}{r}220.7 \\
8.40\end{array}$ & $\begin{array}{c}147.7 \\
9.39\end{array}$ & $\begin{array}{l}82.7 \\
0.15\end{array}$ & $\begin{array}{c}22.5 \\
2.35\end{array}$ & $\begin{array}{c}39.3 \\
2.50\end{array}$ & $\begin{array}{l}6.5 \\
0.41\end{array}$ & 1608 \\
\hline N2 & $b$ & $\begin{array}{c}296.6 \\
13.92\end{array}$ & $\begin{array}{l}591.9 \\
9.01\end{array}$ & $\begin{array}{r}972.2 \\
8.95\end{array}$ & $\begin{array}{c}247.3 \\
1.18\end{array}$ & $\begin{array}{c}87.9 \\
1.04\end{array}$ & $\begin{array}{c}36.6 \\
1.31\end{array}$ & $\begin{array}{l}337.8 \\
6.59\end{array}$ & $\begin{array}{c}509.5 \\
75.12\end{array}$ & $\begin{array}{r}254.7 \\
6.83\end{array}$ & $\begin{array}{l}137.6 \\
1.65\end{array}$ & $\begin{array}{c}38.6 \\
0.92\end{array}$ & $\begin{array}{c}53.9 \\
0.12\end{array}$ & $\begin{array}{l}6.5 \\
0.60\end{array}$ & 3571 \\
\hline B1 & $b$ & $\begin{array}{c}342.9 \\
21.53\end{array}$ & $\begin{array}{l}2021.5 \\
300.94\end{array}$ & $\begin{array}{r}1335.1 \\
16.95\end{array}$ & $\begin{array}{c}454.9 \\
17.27\end{array}$ & $\begin{array}{c}66.9 \\
4.23\end{array}$ & $\begin{array}{c}25.8 \\
0.47\end{array}$ & $\begin{array}{r}313.5 \\
1.21\end{array}$ & $\begin{array}{r}1158.6 \\
54.30\end{array}$ & $\begin{array}{r}333.1 \\
6.47\end{array}$ & $\begin{array}{r}244.9 \\
2.88\end{array}$ & $\begin{array}{c}71.1 \\
1.20\end{array}$ & $\begin{array}{c}84.2 \\
0.22\end{array}$ & $\begin{array}{l}7.1 \\
0.07\end{array}$ & 6460 \\
\hline B2 & b & $\begin{array}{l}554.6 \\
1.11\end{array}$ & $\begin{array}{l}957.5 \\
6.45\end{array}$ & $\begin{array}{c}756.1 \\
10.21\end{array}$ & $\begin{array}{r}317.0 \\
2.85\end{array}$ & $\begin{array}{c}125.2 \\
4.49\end{array}$ & $\begin{array}{c}88.3 \\
3.15\end{array}$ & $\begin{array}{l}398.0 \\
13.83\end{array}$ & $\begin{array}{c}235.0 \\
10.13\end{array}$ & $\begin{array}{c}365.8 \\
14.82\end{array}$ & $\begin{array}{r}248.1 \\
0.86\end{array}$ & $\begin{array}{l}53.6 \\
3.91\end{array}$ & $\begin{array}{c}64.3 \\
0.39\end{array}$ & $\begin{array}{c}11.1 \\
0.47\end{array}$ & 4175 \\
\hline S1 & b & $\begin{array}{c}396.3 \\
28.35\end{array}$ & $\begin{array}{l}820.8 \\
9.43\end{array}$ & $\begin{array}{c}915.2 \\
17.94\end{array}$ & $\begin{array}{l}323.3 \\
16.85\end{array}$ & $\begin{array}{l}87.8 \\
6.10\end{array}$ & $\begin{array}{l}27.3 \\
1.15\end{array}$ & $\begin{array}{c}323.8 \\
19.06\end{array}$ & $\begin{array}{l}372.1 \\
48.07\end{array}$ & $\begin{array}{l}309.0 \\
11.20\end{array}$ & $\begin{array}{c}337.7 \\
14.29\end{array}$ & $\begin{array}{c}43.3 \\
0.59\end{array}$ & $\begin{array}{r}135.1 \\
9.66\end{array}$ & $\begin{array}{l}7.2 \\
0.44\end{array}$ & 4099 \\
\hline S2 & b & $\begin{array}{l}435.7 \\
12.47\end{array}$ & $\begin{array}{l}505.6 \\
17.21\end{array}$ & $\begin{array}{c}805.3 \\
3.42\end{array}$ & $\begin{array}{r}230.4 \\
0.63\end{array}$ & $\begin{array}{c}43.9 \\
0.07\end{array}$ & $\begin{array}{l}35.9 \\
0.45\end{array}$ & $\begin{array}{r}219.2 \\
2.74\end{array}$ & $\begin{array}{l}716.0 \\
23.40\end{array}$ & $\begin{array}{l}272.9 \\
16.47\end{array}$ & $\begin{array}{r}205.0 \\
4.56\end{array}$ & $\begin{array}{c}51.6 \\
2.02\end{array}$ & $\begin{array}{c}83.9 \\
3.85\end{array}$ & $\begin{array}{c}23.0 \\
1.64\end{array}$ & 3629 \\
\hline B1 & c & $\begin{array}{l}281.8 \\
8.89\end{array}$ & $\begin{array}{l}488.5 \\
34.21\end{array}$ & $\begin{array}{c}545.9 \\
41.61\end{array}$ & $\begin{array}{c}173.1 \\
27.87\end{array}$ & $\begin{array}{c}64.9 \\
3.66\end{array}$ & $\begin{array}{c}37.9 \\
1.52\end{array}$ & $\begin{array}{r}234.2 \\
3.13\end{array}$ & $\begin{array}{c}784.3 \\
0.24\end{array}$ & $\begin{array}{r}170.5 \\
2.51\end{array}$ & $\begin{array}{c}89.8 \\
3.77\end{array}$ & $\begin{array}{l}27.7 \\
0.16\end{array}$ & $\begin{array}{l}48.0 \\
1.00\end{array}$ & $\begin{array}{c}27.9 \\
0.80\end{array}$ & 2974 \\
\hline B2 & c & $\begin{array}{r}282.3 \\
4.89\end{array}$ & $\begin{array}{l}787.4 \\
45.40\end{array}$ & $\begin{array}{r}1111.3 \\
86.11\end{array}$ & $\begin{array}{l}329.6 \\
13.90\end{array}$ & $\begin{array}{l}409.0 \\
474.37\end{array}$ & $\begin{array}{l}61.0 \\
4.91\end{array}$ & $\begin{array}{r}271.8 \\
8.82\end{array}$ & $\begin{array}{c}1069.1 \\
52.55\end{array}$ & $\begin{array}{l}149.0 \\
5.46\end{array}$ & $\begin{array}{c}103.7 \\
10.80\end{array}$ & $\begin{array}{c}32.3 \\
0.13\end{array}$ & $\begin{array}{c}88.7 \\
1.66\end{array}$ & $\begin{array}{c}61.2 \\
0.89\end{array}$ & 4756 \\
\hline S1 & c & $\begin{array}{c}374.3 \\
1.21\end{array}$ & $\begin{array}{l}1365.4 \\
17.22\end{array}$ & $\begin{array}{c}1069.4 \\
26.01\end{array}$ & $\begin{array}{c}401.0 \\
0.34\end{array}$ & $\begin{array}{l}164.1 \\
6.06\end{array}$ & $\begin{array}{c}11.3 \\
0.13\end{array}$ & $\begin{array}{c}271.4 \\
1.12\end{array}$ & $\begin{array}{r}1608.0 \\
1.48\end{array}$ & $\begin{array}{l}226.0 \\
6.48\end{array}$ & $\begin{array}{c}197.7 \\
2.47\end{array}$ & $\begin{array}{c}45.5 \\
0.10\end{array}$ & $\begin{array}{l}86.1 \\
15.03\end{array}$ & $\begin{array}{c}60.5 \\
0.51\end{array}$ & 5881 \\
\hline S2 & c & $\begin{array}{c}224.4 \\
20.92\end{array}$ & $\begin{array}{l}117.8 \\
10.73\end{array}$ & $\begin{array}{c}340.8 \\
27.89\end{array}$ & $\begin{array}{c}73.7 \\
7.69\end{array}$ & $\begin{array}{c}42.9 \\
0.41\end{array}$ & $\begin{array}{c}13.7 \\
1.48\end{array}$ & $\begin{array}{l}288.3 \\
25.80\end{array}$ & $\begin{array}{l}213.7 \\
18.44\end{array}$ & $\begin{array}{r}131.1 \\
8.13\end{array}$ & $\begin{array}{l}72.9 \\
10.50\end{array}$ & $\begin{array}{c}12.7 \\
2.06\end{array}$ & $\begin{array}{c}18.3 \\
2.04\end{array}$ & $\begin{array}{l}6.4 \\
0.22\end{array}$ & 1557 \\
\hline & mean & 502.9 & 2355.3 & 1506.9 & 645.4 & 280.8 & 62.8 & 342.5 & 1271.0 & 282.6 & 246.9 & 53.7 & 86.5 & 33.0 & 7567 \\
\hline & $\max$ & 1263.6 & 9749.2 & 5589.6 & 2718.5 & 1413.2 & 288.6 & 911.8 & 5191.2 & 589.8 & 813.5 & 164.4 & 249.3 & 126.6 & 25992 \\
\hline & $\min$ & 224.4 & $\mathrm{nq}$ & 246.3 & 33.2 & 37.0 & $\mathrm{nq}$ & 147.1 & 213.7 & 25.3 & 18.3 & 11.2 & 18.3 & 6.4 & 1557 \\
\hline & SD & 257.3 & 2654.6 & 1313.7 & 672.8 & 344.5 & 62.9 & 156.1 & 1203.1 & 132.0 & 177.6 & 36.8 & 53.1 & 31.7 & 6622 \\
\hline
\end{tabular}

\footnotetext{
${ }^{a}$ Results are expressed as mean of three determinations; italic figures indicate standard deviation. $\Sigma$, sum of the determined phenolic compounds. ${ }^{b}$ Harvesting times:
} a, mid-November 2005; b, mid-December 2005; c, mid-January 2006.

Phenolic Compounds. The quality and quantity of phenolics are influenced by the maturity of the leaves, being different in young and old ones (27). In the case of tronchuda cabbage analyzed samples, the phenolic composition of internal (younger) and external leaves (older) was considerably different in terms of qualitative profile and total amounts, as was observed before $(5,25)$. In the internal leaves were found 15 phenolics, of which 5 were kaempferol derivatives and 10 were cinnamic acid derivatives (Figure 2A). The external leaves were characterized by the presence of 3-p-coumaroylquinic acid and 13 kaempferol derivatives (Figure 2B). Only 3-p-coumaroylquinic acid (1), kaempferol 3-O-sophoroside-7-O-glucoside (2), and kaempferol 3-O-sophoroside (9) were found in both leaves. Kaempferol 3-O-sophoroside-7-O-glucoside (2) was the major compound in both kinds of leaves, kaempferol 3-O-sophoroside (9) being also an important one.

The external leaves total phenolic compounds amount was, in general, much higher than that of the internal ones (Tables 1 and 2). This can be ascribed to the fact that the production of phenolics is usually lower during rapid growth of the younger leaves, increasing significantly thereafter, when the photosyn- 
Table 2. Quantification of Brassica oleracea var. costata External Leaves Phenolic Compounds (Millirams per Kilogram, Dry Basis) ${ }^{a}$

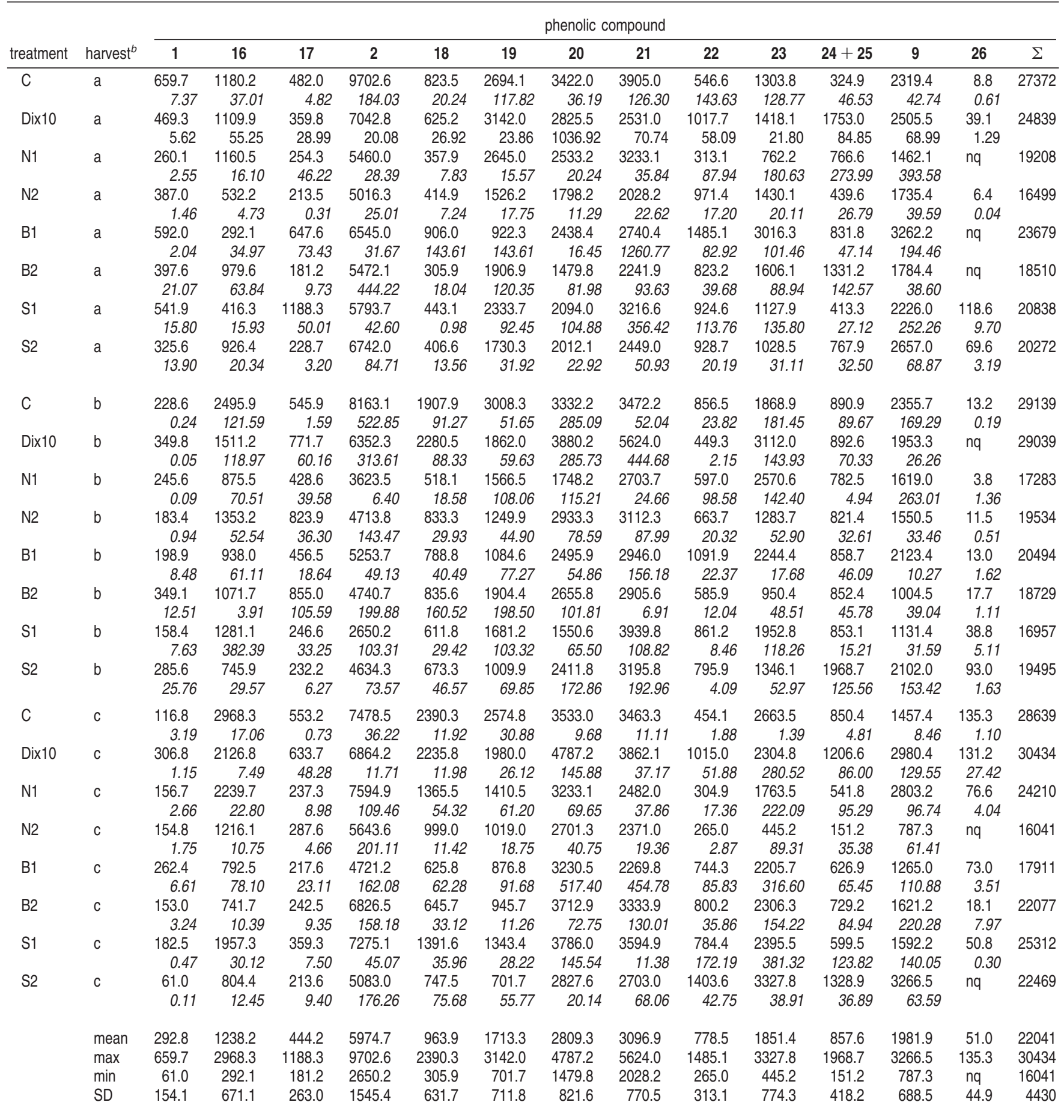

${ }^{a}$ Results are expressed as mean of three determinations; italic figures indicate standard deviation. $\Sigma$, sum of the determined phenolic compounds. ${ }^{b}$ Harvesting times: a, mid-November 2005; b, mid-December 2005; c, mid-January 2006.

thetic capacity of the newly matured leaves is highest and they are no longer growing (18). The kaempferol derivatives present in internal leaves varied between ca. 51 and $90 \%$ of total phenolics. The sinapic acid derivatives represented ca. 7.2-34\%. In this group, sinapic acid (8) was, for most of the samples, the major compound, representing ca. 2.0-18.5\%. The p-coumaroylquinic acid derivatives (compounds $\mathbf{1}$ and 7) varied between 1.7 and $18.5 \%$. Sinapic acid derivatives $\mathbf{1 2}, \mathbf{1 3}+\mathbf{1 4}, \mathbf{1 5}$, and 4- $p$ coumaroylquinic acid (7) were minor compounds in all samples.

As referred to above, external leaves were characterized by the presence of 13 kaempferol derivatives and 3-p-couma- roylquinic acid (compound 1), which represented ca. $0.3-2.6 \%$ of total phenolics. The general pattern of kaempferol glycosylation was substitution in the 3-position with sophoroside, with some compounds simultaneously acylated with cinnamic acids, and in the 7-position with glucose. With the exception of sample $\mathrm{S} 1$, from December 2005 (collection date b), kaempferol 3-Osophoroside-7-O-glucoside (2) was the major compound in all of the samples, accounting for more than ca. $20 \%$ of total phenolics. Kaempferol 3-O-glucoside (26) was found only in very low amounts (less than ca. $0.6 \%$ ). 


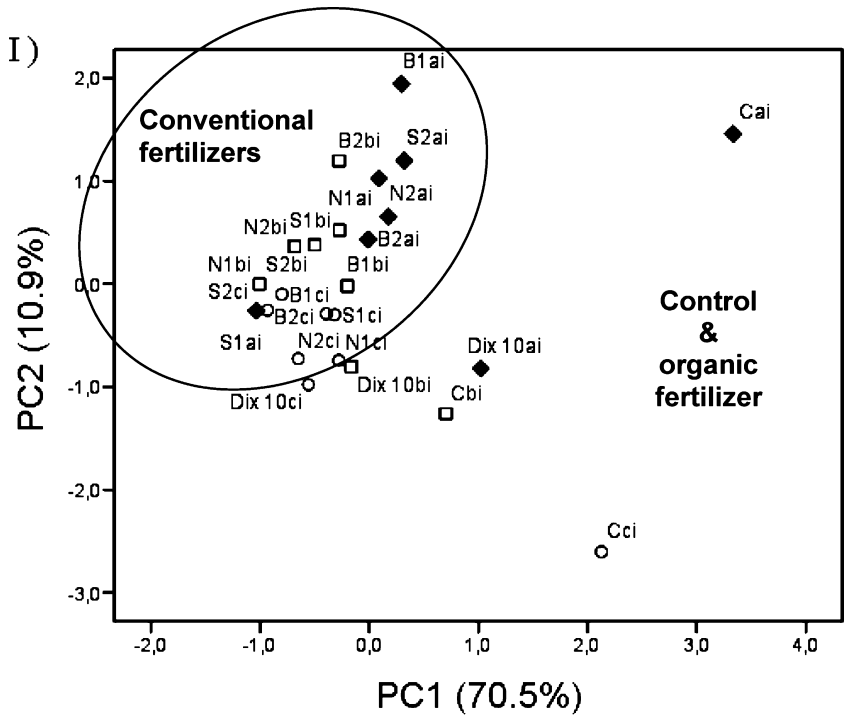

II)

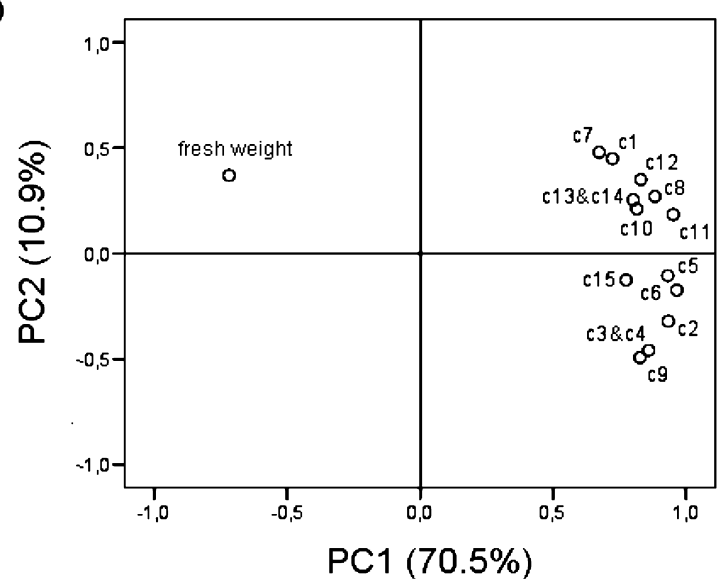

Figure 3. Principal component analysis of phenolic compounds of $B$. oleracea var. costata internal leaves: (I) scores plot; (II) loadings plot. Samples: C, control; Dix 10, organic amendement; N1, $80 \mathrm{~kg}$ of N/ha; $\mathrm{N} 2,160 \mathrm{~kg}$ of N/ha; B1, $2.2 \mathrm{~kg}$ of B/ha; B2, $4.4 \mathrm{~kg}$ of B/ha; $11,37.3 \mathrm{~kg}$ of S/ha; S2, $74.6 \mathrm{~kg}$ of S/ha. Harvesting dates: a, mid-November 2005; b, mid-December 2005; c, mid-December 2006.

General Effects of Fertilization. The patterns of phenolics did not change qualitatively in leaves of $B$. oleracea var. costata grown under different agronomical practices. However, some differences were noticed in quantitative terms (Tables $\mathbf{1}$ and 2). The highest levels of these compounds were detected in control samples, both in internal and in external leaves: external and internal leaves developed without any fertilization contained 1.4- and 4.6-fold more phenolic compounds than the ones that received conventional fertilizer, respectively, and the internal leaves presented 2.4 times more phenolics than the ones grown with organic amendment. Although total phenolics contents were higher in samples with no or organic fertilization, the amounts found per plant, considering the fresh weight, were not very different in all samples. This can be explained by dilution by leaf growth, instead of increased phenolics production (18). Generally, external leaves had a lower dispersion, and this is more evident in terms of the total amount of phenolic compounds, which varied between 16.0 and $30.4 \mathrm{~g} / \mathrm{kg}$ for external leaves and between 1.6 and $26 \mathrm{~g} / \mathrm{kg}$ in the internal ones.

Principal component analysis (PCA) was performed to assess the relationships between phenolic compounds, agronomical practices, and harvesting time. PCA was applied separately to

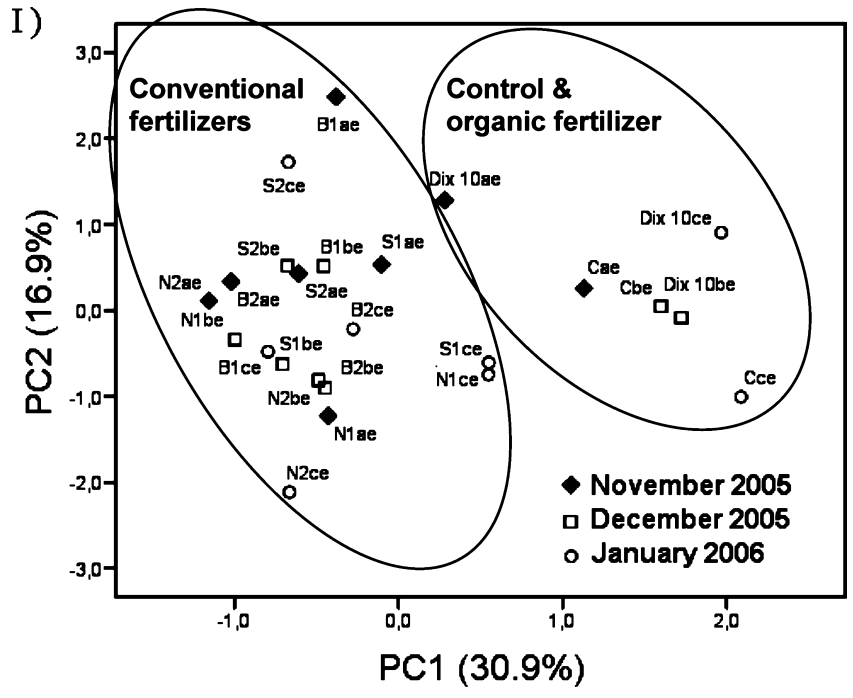

II)

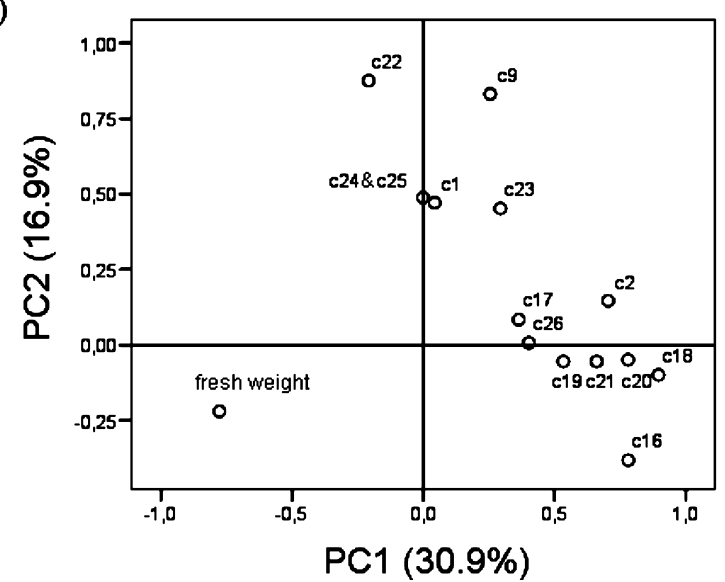

Figure 4. PC1 versus PC2 plot of phenolic compounds of $B$. oleracea var. costata external leaves: (I) scores plot; (II) loadings plot. Identification of the samples is as in Figure 3. Harvesting dates: a, mid-November 2005; b, mid-December 2005; c, mid-December 2006.

both types of leaves due to their qualitative different phenolic profiles, with only three compounds in common, as referred to above (compounds 1, 2, and $\mathbf{9}$ ).

For internal leaves, PCA yielded two PCs (with eigenvalues higher than 1) that accounted for $81.3 \%$ of the total variance in the data (Figure 3). PC1 and PC2 explained 70.5 and $10.9 \%$ of the total variance, respectively. For external leaves (Figures 4 and 5), five PCs with eigenvalues greater than 1 accounted for $79.9 \%$ of the total variance in the data. To simplify the analysis of the results, and considering the scree plot, only the first three PCs were retained, explaining $62.9 \%$ of the total variance $(30.9,16.9$, and $14.2 \%$, respectively).

For both types of leaves (Figures 3 and 4), cabbages produced with organic fertilizer and control were quite effectively separated from those of chemical fertilizations, although they were not clustered according to the amounts and different fertilizers provided in each agronomical practice. Scores of leaves collected in November 2005 (a) fall more separated from those of samples collected in December 2005 (b) or January 2006 (c) (Figures 3 and 5).

Effects of Organic Fertilization. In general, samples from organic fertilization exhibited a tendency for higher total phenolics content than those from conventional practices, collected at the same time (Tables 1 and 2). The higher concentrations of phenolic compounds in those samples can be explained by the increased 


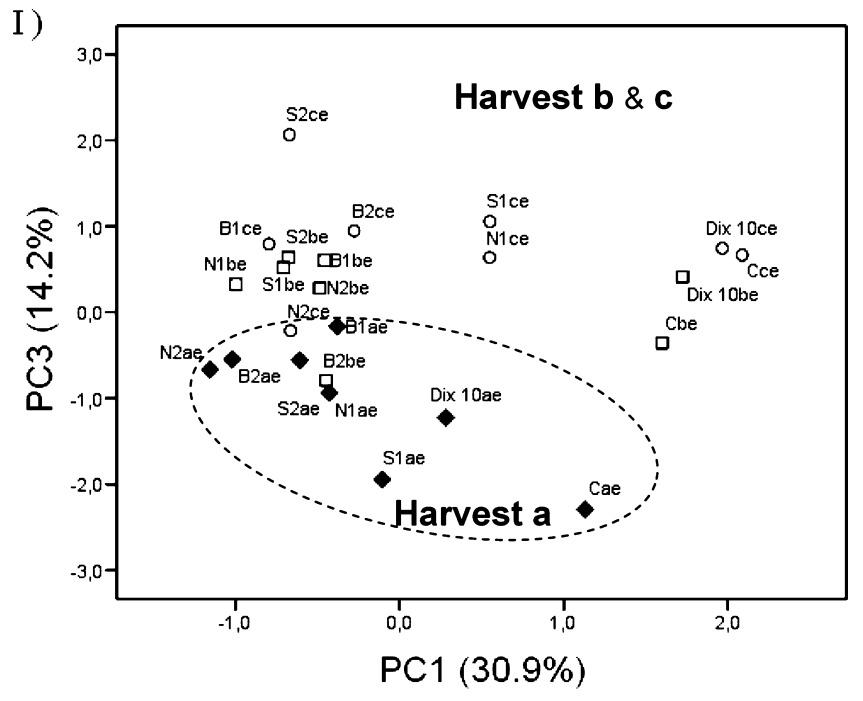

II)

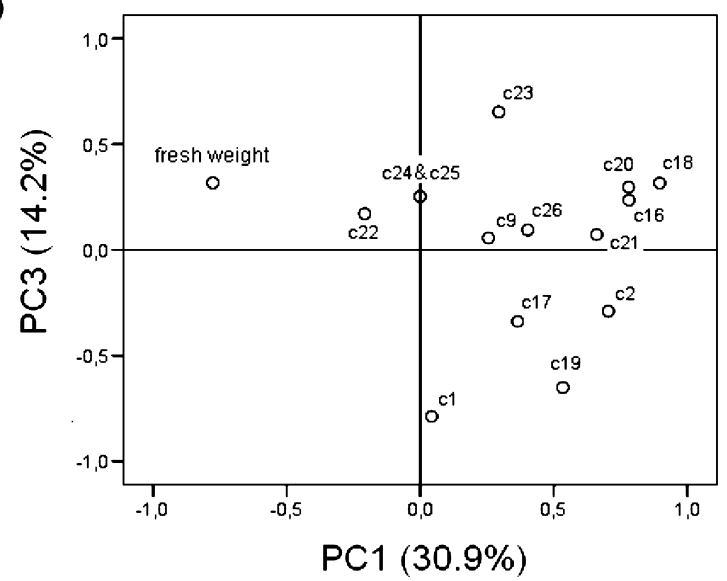

Figure 5. PC1 versus PC3 plot of phenolic compounds of $B$. oleracea var. costata external leaves: (I) scores plot; (II) loadings plot. Identification of the samples is as in Figure 3.

environmental stress caused by the lack of nutrients $(7,8,11,15,23)$, once in this case the degree of mineralization of nitrogen could be insufficient to provide the nutrients needed for the rapid growth of young leaves.

In the internal leaves from organic fertilization, the kaempferol derivatives varied between 79 and $90 \%$ of total phenolics. This proportion decreased in samples from conventional fertilization, in some cases to $50 \%$, being more pronounced in samples fertilized simultaneously with nitrogen and sulfur. It seems that in the internal leaves the organic fertilization induces the acetate/shikimate pathway, resulting in higher production of flavonoids, whereas the conventional fertilization preferentially leads to the shikimate pathway alone, resulting in more phenolic acids. In external leaves the kaempferol derivatives were always higher than $97 \%$.

Effects of Nitrogen Fertilization. Fertilization with nitrogen usually accelerates the vegetative growth of plants, although increased nitrogen availability may decrease the levels of phenolic compounds (14). A trend for increased biomass production (Figure 1) and decreased phenolic compounds concentrations (Tables $\mathbf{1}$ and $\mathbf{2}$ ) was observed with the moderate level of nitrogen (N1 samples). When the results were compared with those obtained with samples treated with higher levels of nitrogen (N2), no relevant differences were found (Figures 3-5).

Low nitrogen availability is the most common growth-limiting factor for primary production of plants $(1,13,14)$. In most crops, yield is clearly improved by nitrogen fertilization, particularly when it is limiting the genetic growth potential $(13,28)$. Maybe with the moderate level of nitrogen the plants reached the genetically determined capacity to form biomass (28). Therefore, these results indicate that it may be possible to obtain good yields in B. oleracea var. costata growth and high phenolics content with minimized nitrogen fertilization. The risk of nitrogen leakage from the soil can thereby be reduced, which has a positive impact on the environment (27).

Effects of Sulfur Fertilization. Application of sulfur together with nitrogen had no significant effects on the growth or phenolics metabolism of B. oleracea var. costata (Figures 1 and 3-5 and Tables 1 and 2). Sulfur assimilation by plants responds dynamically to changes in environmental conditions and to sulfur supply (22). As a consequence, phenolic compounds can vary with sulfur fertilization, due to its effects on phenolic enzymes polyphenol oxidase and peroxidase (29). Yield and quality responses to applied sulfur are likely especially on light sandy soils as atmospheric sulfur inputs are being reduced (21). Maybe in the fields where this experiment was developed, the sulfur inputs, other than the agronomical application, were still enough to ensure good plant development.

Effects of Boron Fertilization. Boron deficiency is the most widespread of all the micronutrient deficiencies (20), although it can be toxic when present in excess (30). The primary effect of boron deficiency is the reduction of cell enlargement in growing tissues (30). Other effects are the cessation of root elongation, reduced leaf expansion, and loss of fertility (31). Boron fertilization of $B$. oleracea var. costata, a hardy crop, did not considerably affect its growth and phenolic composition (Tables 1 and 2 and Figures 3-5).

Effects of Harvesting Time. Plants harvested in November, generally with a higher content of phenolic compounds (Tables 1 and 2), showed a good separation from the samples harvested later (Figures 3 and 5). These results may be explained by UV irradiation: the leaves of these samples developed earlier in the winter, with a larger photoperiod, and it is known that this factor induced flavonoids (particularly kaempferol derivatives) and sinapate esters in Arabidopsis (32) and caused an overall increase in the amount of soluble flavonoids in Brassica napus (33). The same was observed for broccoli, for which, in general, rich sulfur fertilization and longer exposure to sunlight lead to higher concentrations of phenolic compounds (29).

In the case of internal leaves phenolics content, the difference between organic and N1 fertilization found in the last collection was smaller than those observed for the samples collected earlier. Maybe at the date of the last collection the plants are already adapted to the fertilization conditions, leading to smaller difference between these two regimes. Additionally, the internal leaves of sample S1, from the first harvesting date (a), showed a distinct phenolic profile, especially with regard to the major compounds kaempferol 3-O-sophoroside-7-O-glucoside (2), which was not quantifiable, and kaempferol 3-O-sophoroside (9), the percentage of which almost corresponds to the sum of compounds $\mathbf{2}$ and $\mathbf{9}$ in the other samples. Therefore, this sample did not cluster with the other samples from the same date (Figure 3).

In terms of individual phenolics, the concentrations of 3-pcoumaroylquinic (1) and 4- $p$-coumaroylquinic (7) acids were significantly higher in the first harvesting and for all treatments of internal leaves (Figure 3, II).

On the other hand, no important differences were observed in the percentage of kaempferol 3-O-sophoroside (9), a major compound in both kinds of leaves. The kaempferol glycosylation 
with sophorotrioside, which was found only in external leaves, notably increased from the first sampling time to the last sampling.

The differences found for total phenolics composition at the three harvesting dates were not relevant, being not possible to indicate a time for which the harvest of tronchuda cabbage samples with high phenolics contents is favored. These results show that for these compounds the optimum harvest time spans a large period during the winter.

In conclusion, we can say that $B$. oleracea var. costata can be grown with different fertilization regimens (organic or chemical) without affecting the phenolic profile, although the total amount of phenolic compounds is higher under organic practices. Despite being possible to grow the plant without any kind of fertilization, its development will be greatly affected, but no decrease in phenolics biosynthesis will be observed.

By comparison with the application of a moderate dose of nitrogen $(80 \mathrm{~kg} / \mathrm{ha})$, the use of a high nitrogen quantity (160 $\mathrm{kg} / \mathrm{ha}$ ) or the simultaneous fertilization with a moderate nitrogen dose and boron or sulfur does not considerably affect phenolics and biomass of the plants. Therefore, it is possible to grow tronchuda cabbage without excess fertilizers, with highest amounts of phenolics and reduced environment pollution. The effect of fertilization in other essential nutrients cannot be forgotten and deserves to be explored.

\section{LITERATURE CITED}

(1) Brandt, K.; Mølgaard, J. P. Organic agriculture: does it enhance or reduce the nutritional value of plant foods. J. Sci. Food Agric. 2001, 81, 924-931.

(2) Saito, K. Sulfur assimilatory metabolism. The long and smelling road. Plant Physiol. 2004, 136, 2443-2450.

(3) Rosa, E. A. S. Glucosinolates from flower buds of portuguese Brassica crops. Phytochemistry 1997, 44, 1415-1419.

(4) Rosa, E.; Heaney, R. Seasonal glucosinolate variation in protein, mineral and composition of Portuguese cabbages and kale. Anim. Feed Sci. Technol. 1996, 57, 111-127.

(5) Ferreres, F.; Valentão, P.; Llorach, R.; Pinheiro, C.; Cardoso, L.; Pereira, J. A.; Sousa, C.; Seabra, R. M.; Andrade, P. B. Phenolic compounds in external leaves of tronchuda cabbage (Brassica oleracea L. var. costata DC). J. Agric. Food Chem. 2005, 53, 2901-2907.

(6) Sousa, C.; Valentão, P.; Rangel, J.; Lopes, G.; Pereira, J. A.; Ferreres, F.; Seabra, R. M.; Andrade, P. B. Influence of two fertilization regimens on the amounts of organic acids and phenolic compounds of tronchuda cabbage (Brassica oleracea L. var. costata DC). J. Agric. Food Chem. 2005, 53, 9128-9132.

(7) Anttonen, M. J.; Karjalainen, R. O. High-performance liquid chromatography analysis of black currant (Ribes nigrum L.) fruit phenolics grown either conventionally or organically. J. Agric. Food Chem. 2006, 54, 7530-7538.

(8) Anttonen, M. J.; Hoppula, K. I.; Nestby, R.; Verheul, M. L. J.; Karjalainen, R. O. Influence of fertilization, mulch color, early forcing, fruit order, planting date, shading, growing environment, and genotype on the contents of selected phenolics in strawberry (Fragaria $\times$ ananassa Duch.) fruits. J. Agric. Food Chem. 2006, 54, 2614-2620.

(9) Poudel, D. D.; Horwath, W. R.; Lanini, W. T.; Temple, S. R.; Bruggen, A. H. C. Comparison of soil $\mathrm{N}$ availability and leaching potential, crop yields and weeds in organic, low-input and conventional farming systems in northern California. Agric. Ecosyst. Environ. 2002, 90, 125-137.

(10) Malagoli, P.; Laine, P.; Rossato, L.; Ourry, A. Dynamics of nitrogen uptake and mobilization in field-grown winter oilseed rape (Brassica napus) from stem extension to harvest I. Global $\mathrm{N}$ flows between vegetative and reproductive tissues in relation to leaf fall and their residual N. Ann. Bot. 2005, 95, 853-861.
(11) Chassy, A. W.; Bui, L.; Renaud, E. N. C.; Horn, M.; Mitchell, A. E. Three-year comparison of the content of antioxidant microconstituents and several quality characteristics in organic and conventionally managed tomatoes and bell peppers. J. Agric. Food Chem. 2006, 54, 8244-8252.

(12) Rapisarda, P.; Calabretta, M. L.; Romano, G.; Intrigliolo, F. Nitrogen metabolism components as a tool to discriminate between organic and conventional citrus fruits. J. Agric. Food Chem. 2005, 53, 2664-2669.

(13) Cantarero, I. L.; Ruiz, J. M.; Hernandez, J.; Romero, L. Nitrogen metabolism and yield response to increases in nitrogen-phosphorus fertilization: improvement in greenhouse cultivation of eggplant (Solanum melongena cv. Bonica). J. Agric. Food Chem. 1997, 45, 4227-4231.

(14) Witzell, J.; Shevtsova, A. Nitrogen-induced changes in phenolics of Vaccinium myrtillus-implications for interaction with a parasitic fungus. J. Chem. Ecol. 2004, 30, 1937-1956.

(15) Keski-Saari, S.; Julkunen-Tiitto, R. Resource allocation in different parts of juvenile mountain birch plants: effect of nitrogen supply on seedling phenolics and growth. Physiol. Plantarum 2003, 118, 114-126.

(16) Heinaäho, M.; Pusenius, J.; Julkunen-Tiitto, R. Effects of different organic farming methods on the concentration of phenolic compounds in sea buckthorn leaves. J. Agric. Food Chem. 2006, 54, 7678-7685.

(17) Fritz, C.; Palacios-Rojas, N.; Feil, R.; Stitt, M. Regulation of secondary metabolism by the carbon-nitrogen status in tobacco: nitrate inhibits large sectors of phenylpropanoid metabolism. Plant J. 2006, 46, 533-548.

(18) Riipi, M.; Ossipov, V.; Lempa, K.; Haukioja, E.; Koricheva, J.; Ossipova, S.; Pihlaja, K. Seasonal changes in birch leaf chemistry: are there trade-offs between leaf growth and accumulation of phenolics? Oecologia 2002, 130, 380-390.

(19) Blevins, D. G.; Lukaszewski, K. M. Boron in plant structure and function. Annu. Rev. Plant Physiol. Plant Mol. Biol. 1998, 49, 481-500.

(20) Kariot, A.; Chatzopoulou, A.; Bilia, A. R.; Liakopoulos, G.; Stavrianakou, S.; Skaltsa, H. Novel secoiridoid glucosides in Olea europaea leaves suffering from boron deficiency. Biosci., Biotechnol., Biochem. 2006, 70, 1898-1903.

(21) Thomas, S. G.; Hocking, T. J.; Bilsborrow, P. E. Effect of sulfur fertilisation on the growth and metabolism of sugar beet grown on soils of differing sulfur status. Field Crop Res. 2003, 83, 223235.

(22) Leustek, T.; Martin, M. N.; Bick, J. A.; Davies, J. P. Pathways and regulation of sulfur metabolism revealed through molecular and genetic studies. Annu. Rev. Plant Physiol. Plant Mol. Biol. 2000, 51, 141-165.

(23) Young, J. E.; Zhao, X.; Carey, E. E.; Welti, R.; Yang, S. S.; Wang, W. Phytochemical phenolics in organically grown vegetables. Mol. Nutr. Food Res. 2005, 49, 1136-1142.

(24) Vrchovská, V.; Sousa, C.; Valentão, P.; Ferreres, F.; Pereira, J. A.; Seabra, R. M.; Andrade, P. B. Antioxidative properties of tronchuda cabbage (Brassica oleracea $\mathrm{L}$. var. costata DC) external leaves against DPPH, superoxide radical, hydroxyl radical and hypochlorous acid. Food Chem. 2006, 98, 416-425.

(25) Ferreres, F.; Sousa, C.; Vrchovská, V.; Valentão, P.; Pereira, J. A.; Seabra, R. M.; Andrade, P. B. Chemical composition and antioxidant activity of tronchuda cabbage internal leaves. Eur. Food Res. Technol. 2006, 222, 88-98.

(26) Nanda, R.; Bhargava, S. C.; Rawson, H. M. Effect of sowing date on rates of leaf appearance, final leaf numbers and areas in Brassica campestris, B. juncea, B. napu and B. carinata. Field Crop Res. 1995, 42, 125-134.

(27) Mogren, L. M.; Olsson, M. E.; Gertsson, U. E. Quercetin content in field-cured onions (Allium cepa L.): effects of cultivar, lifting time, and nitrogen fertilizer level. J. Agric. Food Chem. 2006, 54, 6185-6191.

(28) Lawlor, D. W. Carbon and nitrogen assimilation in relation to yield: mechanisms are the key to understanding production systems. J. Exp. Bot. 2002, 53, 773-787. 
(29) Vallejo, F.; Barberán, F. A.; Viguera, C. G. Effect of climatic and sulfur fertilisation conditions, on phenolic compounds and vitamin $\mathrm{C}$, in the inflorescences of eight broccoli cultivars. Eur. Food Res. Technol. 2003, 216, 395-401.

(30) Takano, J.; Miwa, K.; Yuan, L.; Wirén, N.; Fujiwara, T. Endocytosis and degradation of BOR1, a boron transporter of Arabidopsis thaliana regulated by boron availability. Proc. Natl. Acad. Sci. U.S.A. 2005, 102, 12276-12281.

(31) Miwa, K.; Takano, J.; Fujiwara, T. Improvement of seed yields under boron-limiting conditions through overexpression of BOR1, a boron transporter for xylem loading, in Arabidopsis thaliana. Plant J. 2006, 46, 1084-1091.
(32) Dixon, R. A.; Paiva, N. L. Stress-induced phenylpropanoid metabolism. Plant Cell 1995, 7, 1085-1097.

(33) Olsson, L. C.; Veit, M.; Weissenböck, G.; Bornman, J. F. Differential flavonoid response to enhanced UV-b radiation in Brassica napus. Phytochemistry 1998, 49, 1021-1028.

Received for review October 16, 2007. Revised manuscript received December 20, 2007. Accepted January 15, 2008. We are grateful to Fundação para a Ciência e Tecnologia (FCT) for financial support of this work (POCI/AGR/57399/2004). M.S.D.-G. is indebted to FCT for her grant (SFRH/BPD/21757/2005).

JF073041O 